\title{
Statistics of the turbulent kinetic energy dissipation rate and its surrogates in a square cylinder wake flow
}

\author{
N. Lefeuvre, ${ }^{\text {a) }}$ F. Thiesset, L. Djenidi, and R. A. Antonia \\ School of Engineering, University of Newcastle, Australia
}

(Received 27 January 2014; accepted 1 September 2014;

published online 22 September 2014)

\begin{abstract}
A numerical simulation based on the lattice Boltzmann method is carried out in the wake of a square cylinder with the view to investigating possible surrogates for the instantaneous turbulent kinetic energy dissipation rate, $\epsilon$, as well as its mean value, $\bar{\epsilon}$. Various surrogate approximations of $\epsilon$, based on local isotropy $\left(\epsilon_{\text {iso }}\right)$, local axisymmetry along the streamwise direction $x\left(\epsilon_{a, x}\right)$ and the transverse direction $y\left(\epsilon_{a, y}\right)$, local homogeneity $\left(\epsilon_{\text {hom }}\right)$, and homogeneity in the transverse plane, $\left(\epsilon_{4 x}\right)$, are assessed. All the approximations are in agreement with $\bar{\epsilon}$ when the distance downstream of the obstacle is larger than about 40 diameters. Closer to the obstacle, the agreement remains reasonable only for $\bar{\epsilon}_{a, x}, \bar{\epsilon}_{\text {hom }}$ and $\bar{\epsilon}_{4 x}$. The probability density functions (PDF) and joint PDFs of $\epsilon$ and its surrogates show that $\epsilon_{4 x}$ correlates best with $\epsilon$ while $\epsilon_{i s o}$ and $\epsilon_{\text {hom }}$ present the smallest correlation. The results indicate that $\epsilon_{4 x}$ is a very good surrogate for $\epsilon$ and can be used for correctly determining the behaviour of $\epsilon$. ㅇ 2014 AIP Publishing LLC. [http://dx.doi.org/10.1063/1.4895637]
\end{abstract}

\section{INTRODUCTION}

The mean turbulent kinetic energy dissipation rate $\bar{\epsilon}$ is a fundamental quantity in turbulent flow studies and is given by

$$
\bar{\epsilon}=2 \nu \overline{s_{i j} s_{i j}},
$$

where $s_{i j}=1 / 2\left(u_{i, j}+u_{j, i}\right)$ is the fluctuating strain rate, $v$ is the kinematic viscosity, $u_{i}$ is the fluctuation part of the velocity in the direction $x$ ( $i=1$, streamwise direction), $y(i=2$, perpendicular to $x$ and the cylinder), $z$ ( $i=3$, parallel to the cylinder axis), $u_{i, j} \equiv \partial u_{i} / \partial x_{j}$ is the derivative of the velocity component $u_{i}$ along the direction $j$ and the overbar represents time and space, along z-direction, averaging. Since the groundbreaking work of Kolmogorov, ${ }^{1,2}$ it is widely recognized that $\bar{\epsilon}$ plays a crucial role in the theoretical study of small-scale turbulence, ${ }^{3}$ such as in spectral analysis and two-point spatial analysis. Further, an accurate estimation of $\bar{\epsilon}$ is essential, for example, in the context of closure models for the turbulent kinetic energy equation.

The estimation of $\bar{\epsilon}$ requires the knowledge of 12 terms. While all of these terms are readily obtained from direct numerical simulation data, only a few can be measured from single-point time signals and using Taylor's hypothesis. Generally, it is the isotropic expression $\bar{\epsilon}_{i s o}$ which is measured to approximate $\bar{\epsilon}$. It represents a considerable simplification to Eq. (1) and is given by

$$
\bar{\epsilon}_{i s o}=15 v \overline{\left(u_{1,1}\right)^{2}} \text {. }
$$

However, while Eq. (2) may be appropriate in many circumstances where local isotropy is approximately satisfied (e.g. homogeneous and isotropic turbulence or HIT, centreline of wakes and jets, at a sufficiently large distance from the wall in pipe, channel and boundary layer flows), this is not the case in general when the anisotropy of the flow is not negligible. For example, Browne et al., ${ }^{4}$

a)Electronic mail: nathan.lefeuvre@uon.edu.au 
who measured nine components of Eq. (1) and inferred the remaining three, showed that $\bar{\epsilon}_{\text {iso }}$ is not satisfied in the far-field of a circular cylinder wake $(C C) ; \bar{\epsilon}_{i s o}$ was about $45 \%$ smaller than $\bar{\epsilon}$. Similar results were obtained by Fabris ${ }^{5}$ and Aronson and Lofdahl. ${ }^{6}$

Another simplification can be made for estimating Eq. (1) if local homogeneity is satisfied. Under this condition and using relation (22) given by Taylor, ${ }^{7}$ the locally homogeneous expression for the dissipation rate is

$$
\bar{\epsilon}_{h o m}=v \overline{\omega^{2}}
$$

where $\overline{\omega^{2}}$ is the enstrophy $\left(\equiv \overline{\omega_{1}^{2}}+\overline{\omega_{2}^{2}}+\overline{\omega_{3}^{2}}\right)$. Note that $\epsilon$ can be written as 8

$$
\epsilon=2 v\left(\omega^{2} / 2-\nabla^{2} p\right) \text {. }
$$

Averaging Eq. (4) leads to Eq. (3), if the flow is homogeneous. In the near ${ }^{9}$ and far fields of the CC wake, the experimental data show that $\bar{\epsilon}_{\text {hom }}$ is an accurate surrogate for $\bar{\epsilon}$. From an experimental viewpoint, this is of great interest because only nine velocity gradients need to be measured to obtain $\overline{\omega^{2}}$ and thus $\bar{\epsilon}_{\text {hom }}$. However, when $\epsilon_{\text {hom }}=v \omega^{2}$ is used to approximate the instantaneous expression for $\epsilon$, the effect of the term $\nabla^{2} p$ in Eq. (4) is ignored.

Local axisymmetry, which is next to isotropy in order of simplicity but which occurs more commonly in practice, can also be invoked for estimating the energy dissipation rate. George and Hussein ${ }^{10}$ were first to write an expression for $\bar{\epsilon}_{a, x}$. If local axisymmetry along the axis $x_{1}$ is assumed then ${ }^{11}$

$$
\bar{\epsilon}_{a, x}=v\left[-\overline{\left(u_{1,1}\right)^{2}}+2 \overline{\left(u_{1,2}\right)^{2}}+2 \overline{\left(u_{2,1}\right)^{2}}+8 \overline{\left(u_{2,2}\right)^{2}}\right] .
$$

Local axisymmetry with respect to the direction $y$ can also be assumed, ${ }^{12}$ which leads to

$$
\bar{\epsilon}_{a, y}=v\left[8 \overline{\left(u_{1,1}\right)^{2}}+2 \overline{\left(u_{1,2}\right)^{2}}+2 \overline{\left(u_{2,1}\right)^{2}}-\overline{\left(u_{2,2}\right)^{2}}\right] .
$$

Finally, a fifth surrogate for the turbulent kinetic energy dissipation rate has been proposed by Zhu and Antonia. ${ }^{13,14}$ These authors used a vorticity probe comprising $4 \mathrm{X}$-probes to measure all the velocity derivatives simultaneously, except $\overline{\left(u_{2,2}\right)^{2}}$ and $\overline{\left(u_{3,3}\right)^{2}}$. The incompressibility condition $u_{1,1}+u_{2,2}+u_{3,3}=0$ led to the following relation:

$$
\overline{\left(u_{2,2}\right)^{2}}+\overline{\left(u_{3,3}\right)^{2}}=\overline{\left(u_{1,1}\right)^{2}}-2 \overline{\left(u_{2,2}\right)\left(u_{3,3}\right)} .
$$

Assuming homogeneity in the transverse $(y-z)$ plane,

$$
\overline{u_{2,2} u_{3,3}}=\overline{u_{2,3} u_{3,2}} \text {. }
$$

Using Eqs. (7) and (8) leads to the following expression:

$$
\begin{aligned}
\bar{\epsilon}_{4 x} & =v\left[\overline{4\left(u_{1,1}\right)^{2}}+\overline{\left(u_{1,2}\right)^{2}}+\overline{\left(u_{1,3}\right)^{2}}+\overline{\left(u_{2,1}\right)^{2}}+\overline{\left(u_{2,3}\right)^{2}}+\overline{\left(u_{3,1}\right)^{2}}+\overline{\left(u_{3,2}\right)^{2}}\right. \\
& \left.+2 \overline{\left(u_{1,2} u_{2,1}\right)}+2 \overline{\left(u_{1,3} u_{3,1}\right)}-2 \overline{\left(u_{2,3} u_{3,2}\right)}\right] .
\end{aligned}
$$

The above $\bar{\epsilon}$-surrogate should strictly be valid if local homogeneity holds. However, both experimental $^{13,15}$ and numerical ${ }^{16}$ data reveal that its range of validity can be extended. For example, in the intermediate field, Thiesset ${ }^{12}$ showed that $\bar{\epsilon}_{i s o}, \bar{\epsilon}_{a, y}$, and $\bar{\epsilon}_{\text {hom }}$ are good candidates for estimating $\bar{\epsilon}$. In the far field of a $C C$ wake, Hao et al. ${ }^{17}$ showed that, near the wake centerline, $\bar{\epsilon}_{i s o}$ was only about $10 \%$ smaller than $\bar{\epsilon}$, as measured by Browne et al. ${ }^{4}$ This contrasts with the measurements of Browne et al. ${ }^{4}$ who, as previously mentioned, found that $\bar{\epsilon}_{i s o}$ is about $45 \%$ smaller that $\bar{\epsilon}$. These inconsistent results highlight the difficulty of measuring $\bar{\epsilon}$ reliably. Even though $\bar{\epsilon}_{\text {iso }}$ may be adequate in many situations, this does not necessarily imply that local isotropy holds. Indeed, in grid turbulence, Antonia et al. ${ }^{18}$ showed that the velocity derivative ratios depart from their isotropic values. However, they remarked that there are compensations among the 12 components of $\bar{\epsilon}$ which 
collectively lead to $\bar{\epsilon}_{i s o}$ being a reasonable approximation for $\bar{\epsilon}$. Similar observations were made in wakes generated by obstacles. ${ }^{19}$

Moreover, the intermittent character of the velocity derivatives may play a role in the instantaneous distributions of surrogates for $\epsilon$. Indeed, in the original K41 framework, ${ }^{1,2}$ the universality of the inertial range was proposed by supposing the latter to be uniquely determined by $\bar{\epsilon}$. However, Landau and Lifshit ${ }^{20}$ was first to comment that the energy dissipation rate has to be considered as a fluctuating quantity, in the same manner as any other fluctuating quantity. Since the fluctuations of $\epsilon$ are likely to be affected by the non-homogeneous large scales, the concept of universality may break down even for small scales. This behaviour, usually referred to as internal intermittency, has led to an explosion in publications over the last 50 years. Attention has been paid mostly to the departure of scaling exponents from the original K41 predictions. However, the effect of fluctuations in the energy dissipation rate due to the large scales has been given much less attention, even though it is the essence of Landau's original comment. Therefore, not only the mean values but also the instantaneous behaviour of the dissipation rate are of major importance in terms of providing some insight into the intermittent nature of turbulence.

While numerical simulation is the most unambiguous tool for assessing the dissipation rate, the universality of turbulence can be only confirmed or invalidated at very high Reynolds numbers. Such high values of Reynolds numbers remain beyond actual computing capabilities and can only be approached using experiments with the shortcoming that only few velocity derivative components are generally measurable and that the spatial resolution of the probe may be inadequate. One may thus need to explore the adequacy of the different surrogates of $\epsilon$ in a range of Reynolds numbers for which there is overlap between experiments and simulations. The most suitable surrogate as inferred from DNS may then be used in experiments at a much Reynolds numbers.

The present work focuses on assessing the performance of the $\epsilon$-surrogates (Eqs. (2), (3), (5), (6), and (9)) in the wake of a square cylinder $(S q C)$ through direct numerical simulations. The emphasis is on the mean as well as instantaneous values of $\epsilon$. The particular choice of the square cylinder is motivated by the fact that this geometry is easier to implement in numerical simulations. In addition, as shown by Thiesset et al., ${ }^{19}$ a $S q C$ generates a more isotropic wake than a $C C$, a screen, a plate and a screen strip. Accordingly, the $S q C$ wake can be considered as an appropriate test case for the above expressions of the $\epsilon$-surrogates. One can, for example, expect a better agreement between $\bar{\epsilon}$ and $\bar{\epsilon}_{i s o}$ for the $S q C$ than for the other wake generators.

The lattice Boltzmann method for simulating the wake flow is first briefly described in Sec. II and calculations are validated in Sec. III. In Secs. IV and V, particular attention is paid to the mean and root mean square values of $\epsilon$. There are compared with their surrogates at three downstream positions $(x=20,60$, and $100 D)$. Surrogates of $\bar{\epsilon}$ are studied either on the centerline or away from the centerline to appraise the effect of the mean shear. Then, the analysis is extended to the instantaneous values of $\epsilon$ in Sec. VI, by considering the probability density function (PDF). To assess more critically the various approximations for $\epsilon$, the joint probability density function (JPDF) between $\epsilon$ and its surrogates is also examined (Sec. VII). Finally, conclusions are drawn in Sec. VIII.

\section{LATTICE BOLTZMANN METHOD}

The direct numerical simulation of the $S q C$ wake is carried out using the lattice Boltzmann method (LBM). Rather than solving the governing fluid equations (Navier-Stokes equations), the LBM solves the Boltzmann equation for the probability distribution function $f_{i}(\vec{x}, t)$ on a lattice. The basic idea of the LBM is to construct a simplified kinetic model that incorporates the essential physics of microscopic average properties, which obey the desired (macroscopic) Navier-Stokes equations. ${ }^{21}$ With a sufficient amount of symmetry of the lattice, the LBM implicitly resolves these latter equations with second-order accuracy. The Boltzmann equation for $f_{i}(\vec{x}, t)$ is given by

$$
\frac{\partial f}{\partial t}+\vec{e} \nabla_{x} f=\Omega
$$


where $\vec{x}$ is the displacement vector, $\vec{e}$ is the particle velocity, and $\Omega$ is a collision operator approximated by the Bhatnagar-Gross-Krook (LBGK) model

$$
\Omega=\frac{1}{\tau}\left(f^{e q}-f\right),
$$

where $f^{e q}$ is an equilibrium particle distribution function and $\tau$ is the relaxation time. The lattice Boltzmann equation (LBE) is then expressed as

$$
\begin{aligned}
f_{i}\left(\vec{x}+e_{i} \Delta t, t+\Delta t\right)-f_{i}(\vec{x}, t) & =\Omega_{i} \\
& =-\frac{1}{\tau}\left(f_{i}(\vec{x}, t)-f_{i}^{e q}(\vec{x}, t)\right),
\end{aligned}
$$

where $\Delta t$ is the time step, $e_{i}(=\Delta x / \Delta t)$ is the velocity of the particle $i$ in the $i$-direction; $f_{i}^{e q}$ is given by

$$
f_{i}^{e q}(\vec{x}, t)=\rho \omega_{i}\left(1+3 e_{i} \vec{u}+\frac{9}{2}\left(e_{i} \vec{u}\right)^{2}-\frac{3}{2} \vec{u}^{2}\right)
$$

where $\omega_{i}$ is the corresponding weights for each $i$-direction and the macroscopic quantities $\rho$ and $\vec{u}$ are the local fluid density and the local fluid velocity vector defined respectively by

$$
\rho=\sum_{i} f_{i} \quad ; \quad \rho \vec{u}=\sum_{i} f_{i} e_{i} .
$$

Using Eq. (14), Eq. (12) returns the Navier-Stokes equation with a second-order accuracy. In the collision term $\Omega_{i}$ (Eq. (11)), $\tau$ is related to the kinematic viscosity $v$ as follows:

$$
v=\frac{2 \tau-1}{6} .
$$

The LBE is discretized on a Cartesian lattice, $\Delta x=\Delta y=\Delta z=1$, and $\Delta t=1$. A detailed description of the LBM can be found in Chen and Doolen ${ }^{22}$ and Succi. ${ }^{23}$ The motivation for the choice of the LBM over the resolution of the Navier-Stokes equations can be briefly summarised as follows: (i) extreme ease of solid surfaces implementation, (ii) no need for solving the Poisson equation for the pressure, and (iii) which follows in part from (ii), the collision is local in nature, making the implementation of parallel computation extremely easy. It was shown that the $L B M$ based on a single relaxation time $(S R T-L B M)$ may have some issues relating to the pressure field that can be addressed via a multiple relaxation time $(M R T-L B M),{ }^{24}$ in particular close to the obstacle and the boundary conditions. However, Nie et al..$^{25}$ showed that the $M R T-L B M$ can also have difficulties in capturing adequately the pressure field. Further, they show that despite the fact that the $S R T-L B M$ may predict the pressure field with some errors, the turbulent kinetic energy and its dissipation rate are well captured using the $S R T-L B M$ simulations and compare well with those obtained using the $M R T-L B M$ simulations. Quite importantly, Luo et al. ${ }^{24}$ showed that if the mesh resolution is sufficient to capture the salient features of turbulent flows then the $S R T-L B M$ performs as effectively as the $M R T-L B M$ in terms of simulating the pressure field. As shown in Sec. III, the mesh resolution used in the present simulation is fine enough to capture not only the small-scale motions but also to resolve the pressure field at a level that it does not impact the turbulence statistics presented. Note that the method was successfully used for simulating turbulent flows. ${ }^{16,26-31}$

\section{VALIDATION OF THE NUMERICAL SIMULATION}

The three-dimensional Cartesian computational domain (Fig. 1) has $110 D \times 20 D \times 10 D$ mesh points in the streamwise $(x)$, across the wake $(y)$, transversal $(z)$ directions, and $D=12$ ( $\mathrm{D}$ is the size of one side of the square section of the obstacle). The mesh increments in the three directions are equal and regular with $\Delta x=\Delta y=\Delta z=1$. The centre of the obstacle is located at $8 D$ from the inlet and $10 D$ from both top and bottom boundaries of the domain. The downstream distance extends to $x / D=100$ where the origin of $x$ is taken at the centre of the cylinder. 


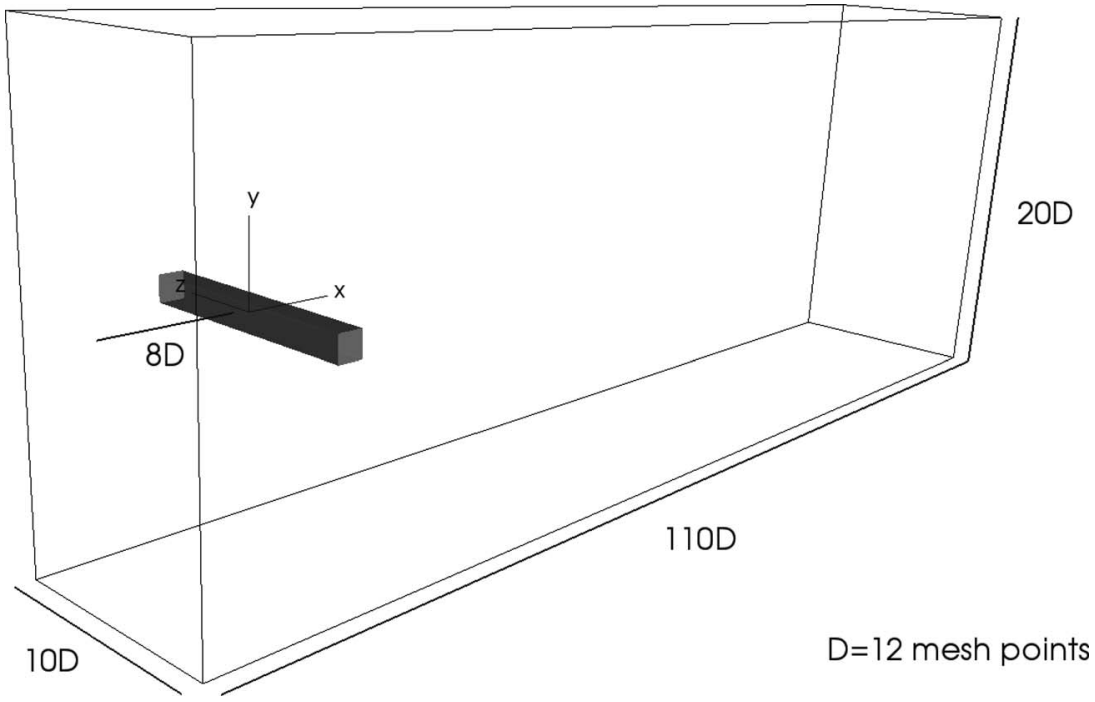

FIG. 1. Unscaled sketch of the computational domain.

Periodic conditions are applied in the $y$ and $z$ directions. At the inlet, a uniform velocity $\left(U_{\text {inlet }}\right.$ $=U_{0}=0.0833$ and $V_{\text {inlet }}=W_{\text {inlet }}=0$ ) is imposed while a convective boundary condition is applied at the outlet of the domain. A no-slip condition at the wall is simulated with a bounce-back scheme, which means that, at the wall, the particles bounce back along the same direction they arrived from. The Reynolds number $R_{D}=U_{0} D / v$ is equal to 2000 . This value is sufficiently small to allow a relatively good spatial resolution and sufficiently large to be comparable with previous experiments.

In order to avoid the occurrence of instabilities where the magnitude of the local strain rate could be large, mainly around the cylinder, a Large Eddy Simulation scheme (LES) based on the Smagorinsky model is used

$$
v_{\text {total }}=v+v_{\tau}
$$

where $v_{\tau}$ is the turbulent viscosity

$$
\nu_{\tau}=(C \Delta)^{2}|S|,
$$

where $C$ is the Smagorinsky constant $(=0.1)$, the cut-off separation $\Delta=\Delta x$ and $S=\sqrt{2 S_{i j} S_{i j}}$ is the magnitude of the strain rate tensor (further details can be found in Djenidi ${ }^{26}$ and Djenidi et al. ${ }^{29}$ ).

An a posteriori check of the spatial resolution reveals that the computational mesh resolution which varies from about $3.5 \eta$ at $x / D \simeq 1$ to $1 \eta$ at $x / D \simeq 100$ (Fig. 2(a)) is comparable to that of many existing DNSs. ${ }^{26,37,38}$ Figure 2(b) compares the present PDF of $\left(\epsilon_{4 x}-\overline{\epsilon_{4 x}}\right)$ with that obtained in a 3D periodic box turbulence simulation, ${ }^{14}$ with a spatial resolution equal to $0.55 \eta$, at $R_{\lambda}=45$ using Eq. (9). The relatively good collapse of the two distributions increases our confidence in the ability of the LBM simulations for capturing the small scales adequately. Note that this collapse supports the quasi-universality of the small-scale motion.

To determine whether the $L E S$ scheme implemented in the $L B M$ impacts on the small-scale results, we report in Figs. 3(a) and 3(b) the velocity spectrum of the longitudinal velocity fluctuation $u$ and its compensated counterpart (i.e., multiplied by $k_{1}^{4}$ ), respectively, at different downstream locations. The profiles are normalized using the Kolmogorov velocity and length scales. We also added the measured spectrum in a cylindrical wake at $x / D=80$ (these measurements are currently being made at the University of Newcastle), and the DNS velocity spectrum on the centreline of a turbulent channel flow. ${ }^{32}$ The figures clearly reveal that the $L E S-L B M$ results compare well with the measurements and DNS in the dissipative ranges showing that the present $L E S-L B M$ captures well the small-scale motion. The $L E S$ scheme does not affect the small-scale motion at scales at least larger than or equal to the mesh resolution. 


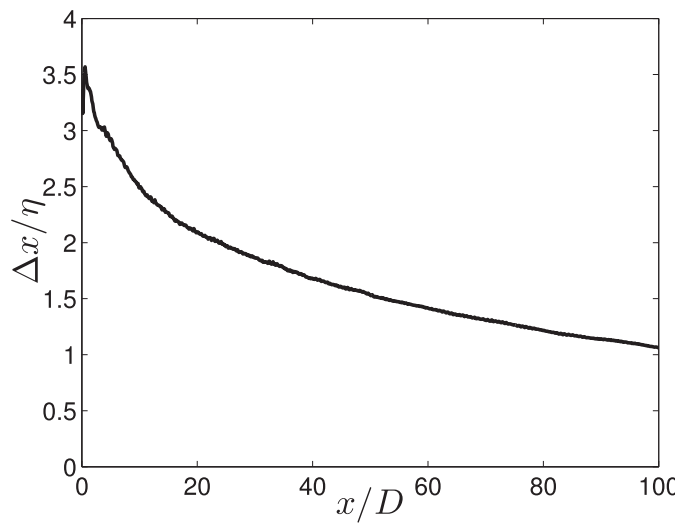

(a)

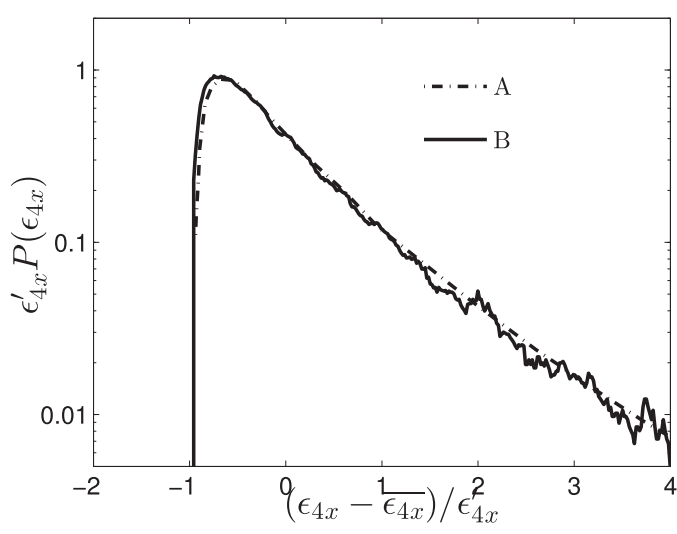

(b)

FIG. 2. (a) Variation of $\Delta x / \eta$ with $x$ along the axis of the $S q C$ wake. (b) Probability density function of the turbulent kinetic energy dissipation inferred from Eq. (9) at $x / D=100$ on the centerline. Solid line: present data (SC); dashed dotted line: DNS from Antonia et al. ${ }^{14}$ using Eq. (9).

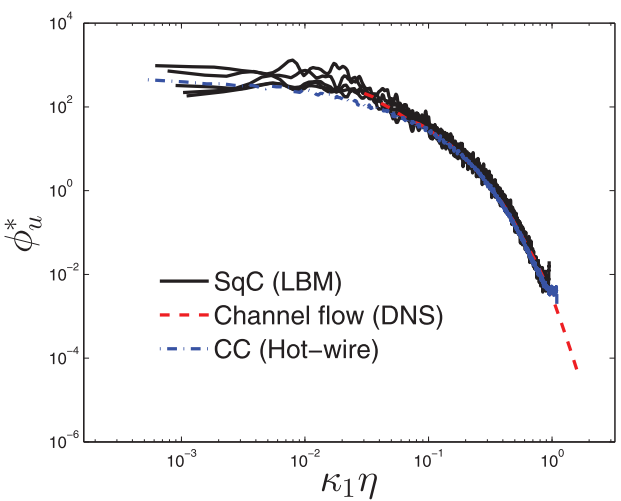

(a)

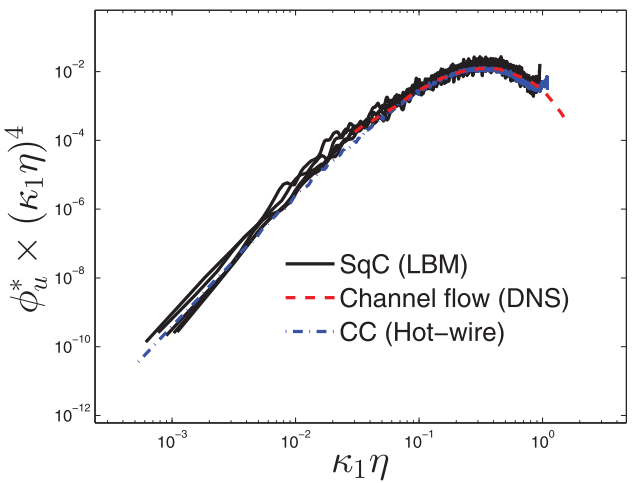

(b)

FIG. 3. (a) Spectra of $u$ and (b) $\phi_{u}^{*} k^{* 4}$ versus $\kappa \eta$. - : axis of $S q C$ at $x / D=30-90(L B M) ;-\cdot-$ : axis of CC at $80 D$ (hot wire, these measurements are currently being made at the University of Newcastle) ; - - - centerline data in a channel flow $\left(\mathrm{DNS}^{32}\right.$ ). Kolmogorov scaling, $\eta$ and $U_{k}$, have been used for normalization.

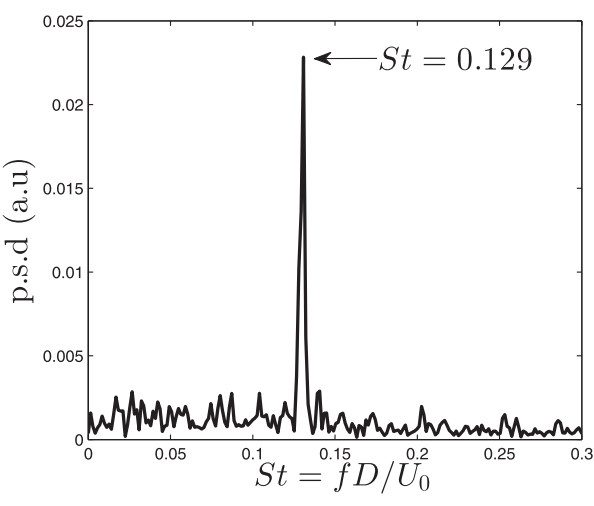

(a)

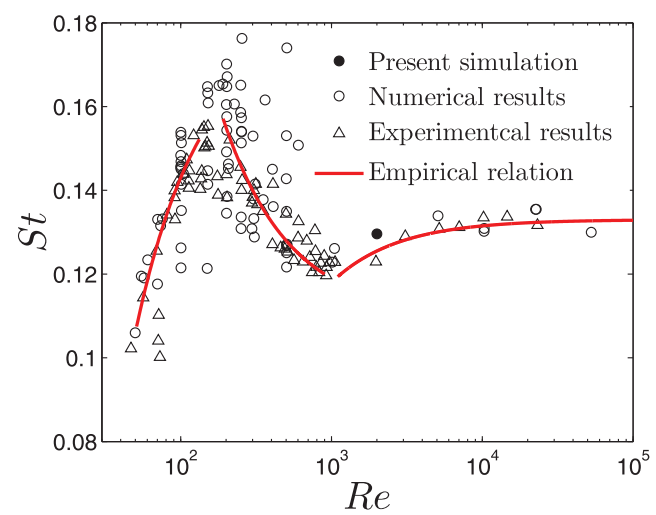

(b)

FIG. 4. (a) Spectra of $\epsilon$ at $x / D=20$ and $y / D=4$. (b) Variation of the Strouhal number with the Reynolds number. Experimental results, ${ }^{33-36}$ Numerical results. ${ }^{36}$ 


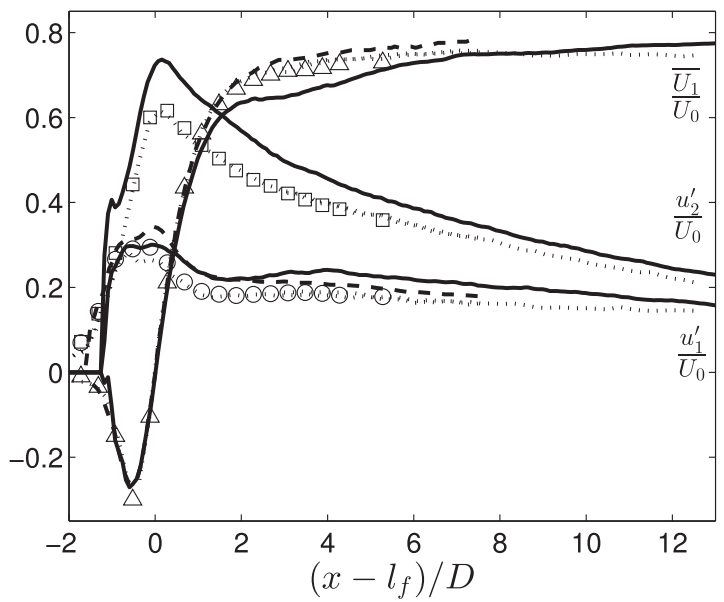

FIG. 5. Streamwise distributions of $\overline{U_{1}} / U_{0}, u_{1}^{\prime} / U_{0}$, and $u_{2}^{\prime} / U_{0}$, on the centerline of a $C C$ wake. Solid line: LBM; dashed line: LES; dotted line: PIV; ${ }^{12}$ symbols: ADL ${ }^{12}$ measurements.

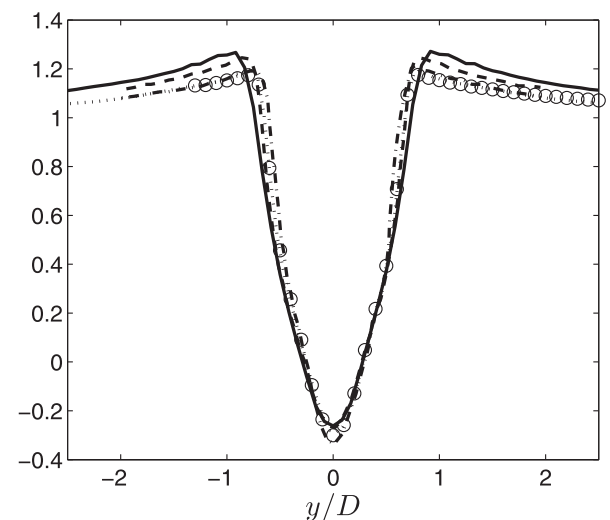

(a) $\overline{U_{1}} / U_{0}$

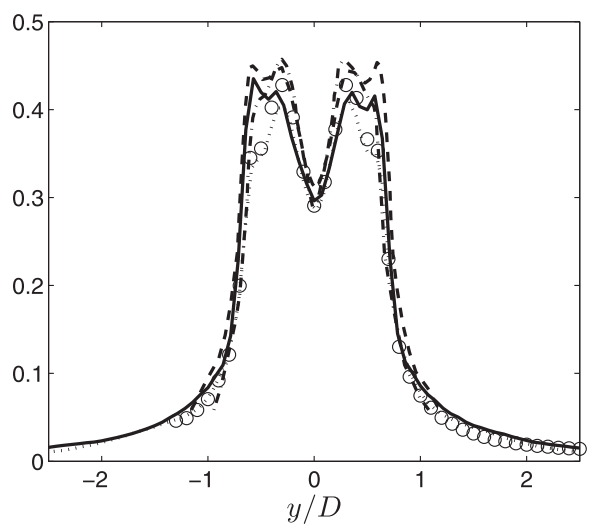

(c) $u_{1}^{\prime} / U_{0}$

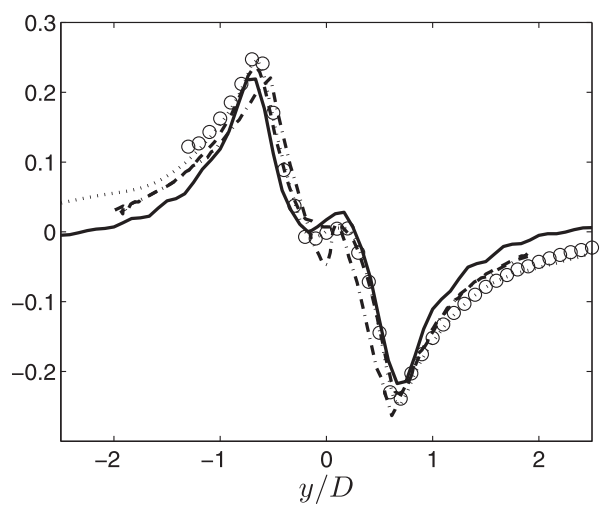

(b) $\overline{U_{2}} / U_{0}$

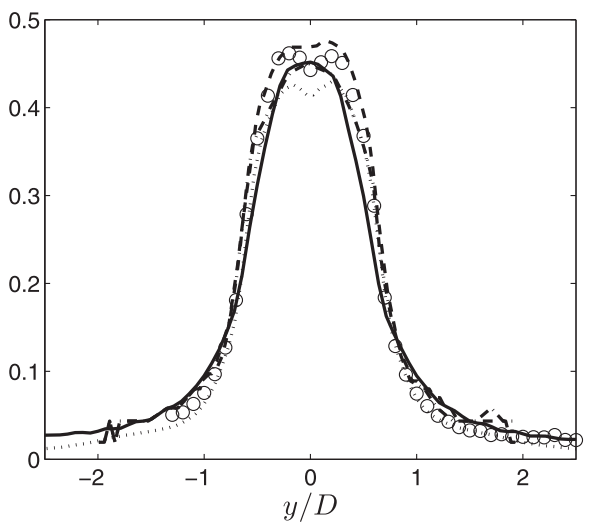

(d) $u_{2}^{\prime} / U_{0}$

FIG. 6. Profiles of $\overline{U_{1}}$ (a), $\overline{U_{2}}$ (b), $u_{1}^{\prime}$ (c), and $u_{2}^{\prime}(\mathrm{d})$ at $\left(x-l_{f}\right) / D=0.54$. Solid line: LBM; doted line: PIV; ${ }^{12}$ dashed-dotted line: DNS $;^{38}$ dashed line: LES ${ }^{39}$ symbols: Laser Doppler Anemometry measurements (LDA). ${ }^{12}$ 
The flow visualisations (not presented here) showed the classical shedding of the Bénard-von Kármán vortices, with a Strouhal number $S t=f D / U_{0}=0.129$ ( $f$ is the shedding frequency) as shown in Fig. 4. This value of $S t$ is remarkably close to the experimental data of Okajima ${ }^{34}$ who found $S t$ $=0.13$ in a $S q C$ wake at $R e=2000$.

To further assess the validity of the LBM simulation, we compare some of our results with existing experimental and numerical results obtained in the wake of a circular cylinder. Because there are fewer data for a $S q C$ than a $C C$, we carried out a LBM simulation of a $C C$ wake. Figure 5 shows the normalized streamwise distributions of the mean velocity $\overline{U_{1}}$, the velocity fluctuations, $u_{1}^{\prime}$ and $u_{2}^{\prime}$ (the prime denotes the rms value) on the wake centerline; the normalization is based on the length of the recirculation zone behind the cylinder, $l_{f}$, and the incoming velocity $U_{0}$. It has been shown that $l_{f}$ is strongly dependent on the initial conditions. Also $l_{f}$ decreases with increasing Reynolds number. Thiesset ${ }^{12}$ showed that, using $l_{f}$ for the normalization of the downstream position, the profile of $\overline{U_{1}}$ collapses well for different $R e$. The LBM results are consistent with particle image velocimetry $(P I V)$, Laser Doppler velocimetry $(L D V),{ }^{12}$ and LES $^{38}$ results at similar Reynolds numbers. For example, both the location and magnitude of the minimum value of $\overline{U_{1}}$ are well reproduced by the LBM. Also, the present LBM values of $u_{1}^{\prime}$ are in relatively good agreement with the measurement and LES data. However, there are some discrepancies between LBM and measured values of $u_{2}^{\prime}$. While LBM predicts correctly the location of the maximum of $u_{2}^{\prime}$, the magnitude is overpredicted. This is yet to be explained, but the differences may be in part attributed to some differences in the initial conditions of both experiments and simulations. Figure 6 shows the profiles of $\overline{U_{1}}, \overline{U_{2}}, u_{1}^{\prime}$, and $u_{2}^{\prime}$ at $x / D=0.54$. The LBM data are in good agreement with published data ${ }^{12,38,39}$ and capture well all features.

Altogether, the results indicate that the LBM simulation is adequate and reliable for investigating the energy dissipation rate behaviour in the wake of a cylinder.

\section{MEAN VALUES OF THE KINETIC ENERGY DISSIPATION RATE}

The adequacy of the surrogates for estimating the kinetic energy dissipation rate is first investigated at the level of mean quantities. To this end, the distribution of $\bar{\epsilon}$ and its surrogates at $x / D$ $=20,60$, and 100 as a function of the transverse distance are displayed in Figure 7. The normalization is based on the velocity defect, $U_{d}$, and the half-width of the wake, $y_{0.5}$.

All distributions collapse well for $x / D=60$ and 100 indicating that all the surrogates are adequate at these locations. However, this is no longer the case at $x / D=20$ where the collapse excludes $\bar{\epsilon}_{i s o}$ and $\bar{\epsilon}_{a, y}$. In particular, $\bar{\epsilon}_{i s o}$ and $\bar{\epsilon}_{a, y}$ deviate significantly from the other distributions in the region $y / D \leq 1$. It is likely that the constraints imposed by these two expressions on the components of $\bar{\epsilon}$ are not satisfied in this region of the wake, not even on the wake centerline.

The departure of $\bar{\epsilon}_{i s o}$ from $\bar{\epsilon}$ at $20 D$ is most probably caused by the anisotropy of the flow. To assess this issue, the velocity derivatives are compared to Eq. (1) and the results reported in Fig. 8 , which shows the ratios,

$$
\begin{aligned}
K_{1} & =(15 v) \overline{u_{1,1}^{2}} / \bar{\epsilon}, \\
K_{2} & =\frac{1}{2}(15 v) \overline{u_{1,2}^{2}} / \bar{\epsilon}, \\
K_{3} & =\frac{1}{2}(15 v) \overline{u_{1,3}^{2}} / \bar{\epsilon},
\end{aligned}
$$

for $x / D=20,60$, and 100 , respectively; these ratios should be equal to one if isotropy is satisfied. On the wake centerline, $K_{1}$ is larger than unity while $K_{2}$ is smaller and $K_{3}$ is close to 1 for $x / D=20$. At $x / D=60$ and 100, $K_{1}$ and $K_{3}$ agree relatively well with local isotropy on and off the centerline. The ratio $K_{2}$ approaches the isotropic value for $x / D=60$; it is 1 at $100 D$. Figure 8 indicates that the level of anisotropy decreases as $x / D$ increases. Consequently, $\bar{\epsilon}_{i s o}$ differs markedly from $\bar{\epsilon}$ at $x / D=20$. It is remarkable that $\bar{\epsilon}_{i s o}$ approximates $\bar{\epsilon}$ at $x / D=60$, considering the anisotropy of large scales in the 


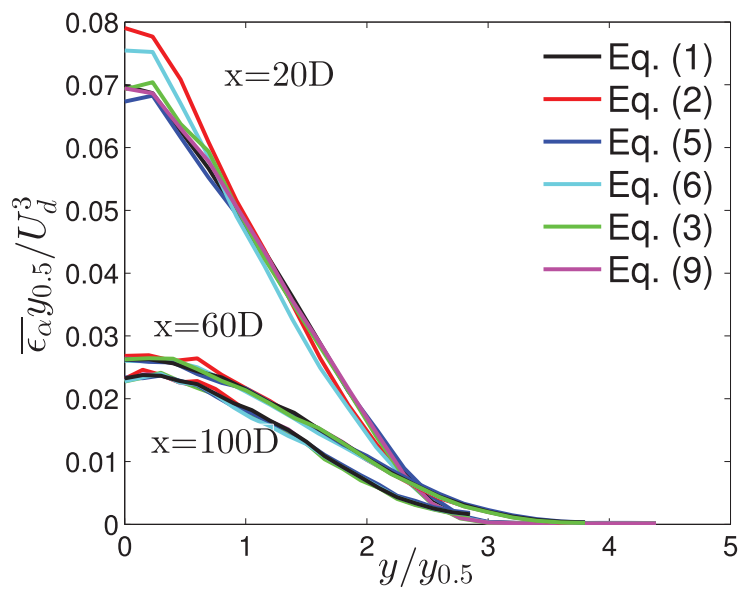

FIG. 7. Comparison of the normalized mean turbulent kinetic energy dissipation rate across the wake.

flow, as illustrated by the departure of $K_{2}$ from 1 at all locations and across the wake width. Using three-component vorticity measurements, Antonia et al. ${ }^{18}$ showed that there is a compensation effect in grid turbulence which may provide some insight into the equality $\bar{\epsilon} \simeq \bar{\epsilon}_{i s o}$. These compensations appear to be at play also in the $S q C$ wake flow in agreement with Ref. 19.

In Ref. 18, the authors also noted satisfactory agreement between $\bar{\epsilon}$ and $\overline{\epsilon_{4 x}}$, which was attributed to the fact that relation (8) was satisfied. Unpublished DNS data in a turbulent channel flow ${ }^{32,40}$ by $\mathrm{Abe}^{41}$ indicate that Eq. (8) is closely satisfied for $y / h \geq 0.1$ ( $y$ is the distance to the wall and $h$ is the half-width of the channel). Figure 9 shows that Eq. (8) is also valid in the present flow, in particular for $x / D=60$ and 100. Clearly, the good collapse observed between $\bar{\epsilon}$ and its surrogates in the present flow shows that compensations are likely to be at play here, in particular away from the centerline where the departure from isotropy becomes important.

Although we cannot comment on the behaviour of $\bar{\epsilon}$ and its surrogates in the far wake, it is worth briefly recalling previously published results. As stated in the introduction, Browne et al. ${ }^{4}$ found that the measured $\bar{\epsilon}_{\text {iso }}$ departed significantly (about $45 \%$ on the wake centerline and about $80 \%$ near the wake edge) from $\bar{\epsilon}$ obtained by measuring nine of its terms and assuming isotropy to infer the remaining three terms. They argued that this latter assumption is unlikely to be critical

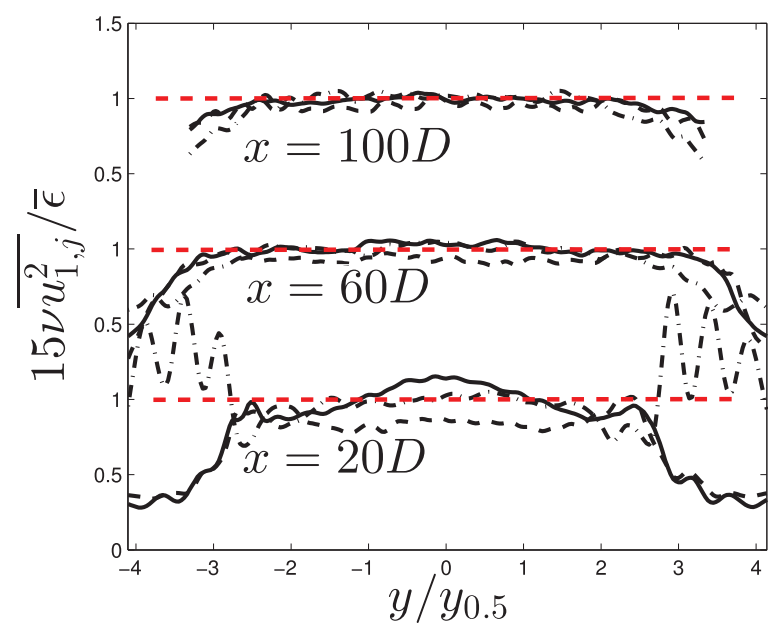

FIG. 8. Distributions across the wake of the ratios $K_{j}$ at $x / D=20,60$, and $100 .-, j=1 ;--, j=2 ;-\cdot-, j=3$; red dashed line, isotropic value of 1 . 


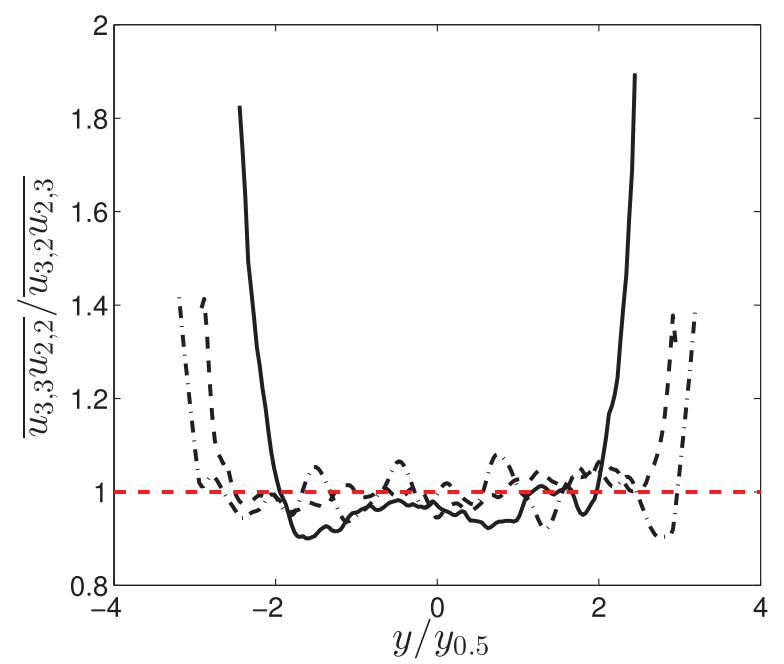

FIG. 9. Distributions across the wake of $\overline{u_{2,2} u_{3,3}} / \overline{u_{2,3} u_{3,2}}$ involved in Eq. (8) at $x=20 D(-), 60 D(---)$, and $100 D$ $(-\cdot-)$; red dashed line, isotropic value.

as they expected the magnitude of those terms to be small by comparison with the other nine. The reasonable closure of their turbulent kinetic energy budget suggests that they measured $\bar{\epsilon}$ with adequate accuracy. However, Hao et al. ${ }^{17}$ measured $\bar{\epsilon}_{a p}=v\left(6 \overline{u_{1,1}^{2}}+3 \overline{u_{1,2}^{2}}+2 \overline{u_{2,1}^{2}}+2 \overline{u_{1,2} u_{2,1}}\right)$ (they assumed local isotropy and homogeneity, and the continuity equation) and observed that $\bar{\epsilon}_{i s o}$ was only about $6 \%$ smaller than $\bar{\epsilon}_{a p}$ across the wake at $x / D=420$ ). Unfortunately, these authors did not verify whether or not the measured $\bar{\epsilon}_{a p}$ closed the turbulent kinetic energy budget. Clearly, the discrepancy between the results of Browne et al. ${ }^{4}$ and those of Hao et al. ${ }^{17}$ requires further investigation, preferably via DNS. It should be noted however that whereas Browne et al. estimated the mean squared values of $u_{i, j}$ from 2-point correlations as $r \rightarrow 0$ ( $r$ is the spatial separation), Hao et al. ${ }^{17}$ applied spectral corrections based on local isotropy, which may have introduced a systematic bias in their results and led to the close agreement between $\bar{\epsilon}_{i s o}$ and $\bar{\epsilon}$.

\section{STANDARD DEVIATION OF THE MEAN KINETIC ENERGY DISSIPATION RATE}

As far as the fluctuating quantities are concerned, one can, for example, investigate the distributions of the standard deviation of $\epsilon, \epsilon^{\prime}=\sqrt{\overline{(\epsilon-\bar{\epsilon})^{2}}}$, across the wake. These are reported in Fig. 10 for $x=20,60$, and 100D. Here again, use was made of appropriate combinations of the velocity defect $U_{d}$ and the half-width $y_{0.5}$ to normalize these quantities.

Noticeable is the fact that, independently of $x$ and the surrogate under consideration, the standard deviation of $\epsilon$ is of same magnitude as its mean value. This indicates that the dissipation rate is a highly fluctuating quantity, in agreement with Landau's comment. This remark is of major importance in the context of refining the theoretical description of turbulence that may therefore account for the fluctuating nature of the dissipation rate.

A careful analysis of Fig. 10 reveals that, irrespectively of the downstream position, $\epsilon^{\prime}$ is relatively well approximated by $\epsilon_{4 x}^{\prime}$, implying that the best candidates appear to be the two axisymmetric expressions, Eqs. (5) and (6), followed by the homogeneous surrogate, Eq. (3), which altogether reveal a maximum departure of about $40 \%-50 \%$ from the real values. Finally, the use of the isotropic expression, Eq. (2), systematically overestimates the standard deviation of $\epsilon$ by almost a factor 2 .

In summary, while $\epsilon_{4 x}$ appears to be the best candidate for capturing the fluctuations of $\epsilon$, the use of the isotropic surrogate leads to some drastic errors. This is particularly important when internal intermittency corrections which account for the fluctuating nature of the dissipation rate have to 
be applied. This is a remarkably challenging task since $\epsilon_{4 x}$ requires measurements using a probe consisting of at least $4 \mathrm{X}$-wires.

It is worth mentioning the fact that the standard deviation of $\epsilon_{4 x}$ is in close agreement with that of $\epsilon$ does not necessarily imply that their respective probabilistic distribution are similar. In Sec. VI, we turn our attention to this issue.

\section{PROBABILITY DENSITY FUNCTIONS}

The results of the preceding Secs. IV and V showed that the energy dissipation rate can be relatively closely approximated by some of its surrogates when mean quantities are considered. However, major departures were observed when the focus is on the standard deviation of $\epsilon$. This is of significant importance from an experimental point of view since $\bar{\epsilon}$ can be approximated adequately from a limited set of measurements, whereas $\epsilon^{\prime}$ requires more complex measurements.

The results in Secs. IV and V do not provide any information on how the instantaneous values of the surrogates compare with $\epsilon$. Some of this information can be obtained through the probability density function (PDF), shown in Figure 11. The normalization of the PDF is such that $\int_{-\infty}^{\infty} P_{\alpha} d \alpha$ $=1$, where $P_{\alpha}$ is the PDF of $\alpha$, and $\alpha$ represents $\epsilon$ and its surrogates.

All the PDFs exhibit a similar positive tail with a nearly exponential form,

$$
P_{\alpha} \sim \exp (-\beta \alpha)
$$

where the constant $\beta$ is estimated to be about 1.25. However, significant differences are visible for $\alpha$ $\leq 0$ at all $x / D$ positions. For example, all the surrogate $P D F$ distributions depart from the PDF of $\epsilon$ at $x / D=20$ and 60. Similar results were observed in grid turbulence ${ }^{18}$ and a turbulent plane wake. ${ }^{13}$ This suggests that the error that one makes using surrogates of $\epsilon$ will be on the most probable values of $\epsilon$ and are therefore of critical importance.

Of all the PDF surrogates for $P_{\epsilon}$, only $P_{\epsilon_{4 x}}$ closely follows $P_{\epsilon}$; the agreement between the two is satisfactory at $x / D=100$. This indicates that $\epsilon_{4 x}$ captures well all instantaneous events ranging from weak to high intensity, and explains the good agreement observed earlier not only between their mean values (Fig. 7) but also between their rms distributions (Fig. 10). This may not be too surprising because 10 out of 12 terms of $\epsilon$ are involved in $\epsilon_{4 x}$. One can notice that the agreement between the surrogates and $\epsilon$ decreases as the number of terms in the surrogate reduces. This remark holds irrespectively of the transverse distance $y$, suggesting that there is no particular effect of the mean shear on the PDFs.

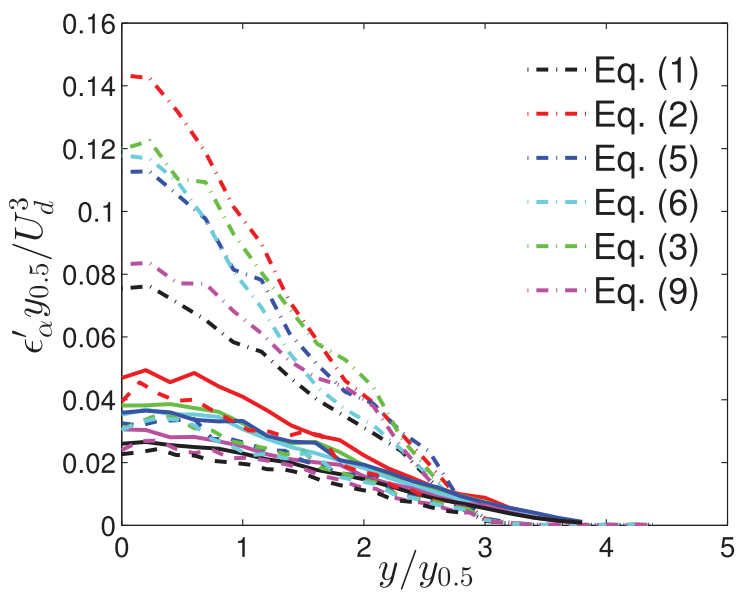

FIG. 10. Comparison of the root mean square energy dissipation rate across the wake. $-\cdot-, x / D=20 D ;-, 60 D ;---$, $100 D$. 


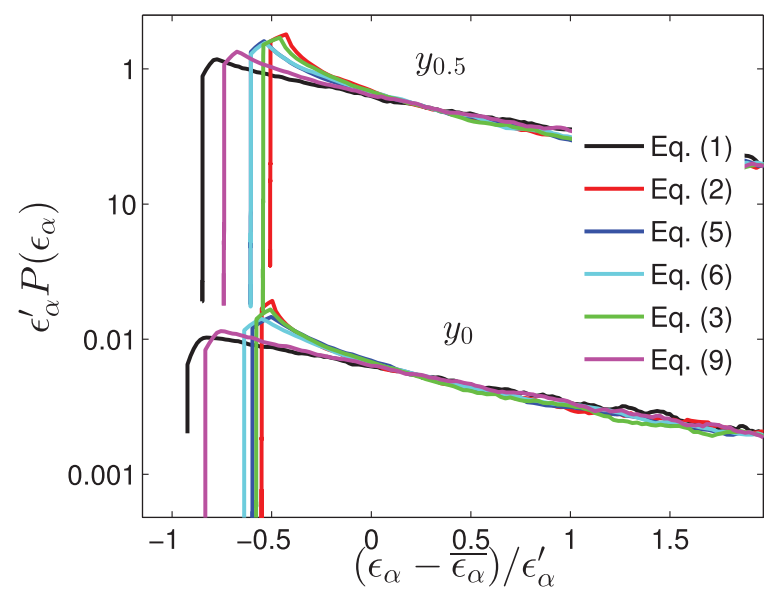

FIG. 11. The probability density function of the energy dissipation rate at two different transverse positions $y=0$ and $y_{0.5}\left(P\left(\epsilon_{\alpha}\right) \times 100\right)$ at $x / D=100$. Black: $\epsilon$; red: $\epsilon_{i s o}$; blue: $\epsilon_{a, x}$; light blue: $\epsilon_{a, y}$; green: $\epsilon_{\text {hom }}$; purple: $\epsilon_{4 x}$.

\section{JOINT PDFs}

A more stringent test of the adequacy of the surrogates for estimating instantaneous values of $\epsilon$ can be further assessed using the joint $P D F$. Indeed, a joint $P D F$ highlights the probability to observe a particular value of $\epsilon$ inferred from one particular surrogate knowing the real value of $\epsilon$. The use of joint $P D F s$ is thus likely to be a more severe test of the adequacy of such surrogates, e.g., some caution is warranted. Indeed, calculating the fluctuations of the various surrogate of $\epsilon$ with the expressions (2), (3), (5), (6), and (9) in which the different terms are not averaged is not strictly correct as these expressions are based on averaged velocity derivatives. The agreement between $P_{\epsilon}$ and $P_{\epsilon_{4 x}}$ is well reflected in the JPDFs of $\epsilon$ and $\epsilon_{4 x}$ shown in Figure 12, which also shows the JPDFs between $\epsilon$ and the other surrogates. These JPDFs are obtained on the centerline of the cylinder wake at $x / D=100$. A perfect correlation between two variables $\alpha_{1}$ and $\alpha_{2}$ would be represented by a straight line $\alpha_{1}=\alpha_{2}$ inclined at $45^{\circ}$. Although none of the JPDFs displays a perfect correlation, the JPDF isocontours of $\epsilon$ and $\epsilon_{4 x}$ indicate the best alignment along a $45^{\circ}$ direction highlighting the reasonable agreement between $P_{\epsilon}$ and $P_{\epsilon_{4 x}}$.

Moreover, there is some degree of correlation between $\epsilon$ and its other surrogates. This can be further assessed from the data of Table I, which reports the correlation coefficients between $\epsilon$ and its surrogates $\left(\rho_{\epsilon, \alpha}, \alpha\right.$ represents any of the surrogates) at $x / D=100$ on the centerline and half-width location. As expected, $\rho_{\epsilon, \epsilon_{4 x}}$ is the largest. Antonia et al. ${ }^{18}$ measured a coefficient $\rho_{\epsilon, \epsilon_{\text {hom }}}$ of about 0.8 in grid turbulence. They argued that a larger value would have been expected had they been able to correct the instantaneous fluctuations for spatial resolution. The present value $(=0.63)$ of $\rho_{\epsilon, \epsilon_{\text {hom }}}$ is not as large as that in grid turbulence. This indicates that the correlation between the energy dissipation rate and enstrophy fluctuations is weaker in the wake than in grid turbulence. In other words, this highlights a stronger effect of pressure fluctuations in the inhomogeneous wake flow by comparison to homogeneous grid turbulence. Table I also shows that $\rho_{\epsilon \epsilon_{\text {hom }}}$ is slightly smaller

TABLE I. Correlation coefficient $\rho_{\epsilon \epsilon_{i s o}}, \rho_{\epsilon \epsilon_{a, x}}, \rho_{\epsilon \epsilon_{a, y}}, \rho_{\epsilon \epsilon_{h o m}}$, and $\rho_{\epsilon \epsilon_{4 x}}$ at $x / D=100$.

\begin{tabular}{lcc}
\hline \hline & $y=0$ & $y_{0.5}$ \\
\hline$\rho_{\epsilon \epsilon_{i s o}}$ & 0.68 & 0.68 \\
$\rho_{\epsilon \epsilon_{a, x}}$ & 0.76 & 0.67 \\
$\rho_{\epsilon \epsilon_{a, y}}$ & 0.76 & 0.78 \\
$\rho_{\epsilon \epsilon_{\text {hom }}}$ & 0.63 & 0.61 \\
$\rho_{\epsilon \epsilon_{4 x}}$ & 0.87 & 0.85 \\
\hline \hline
\end{tabular}




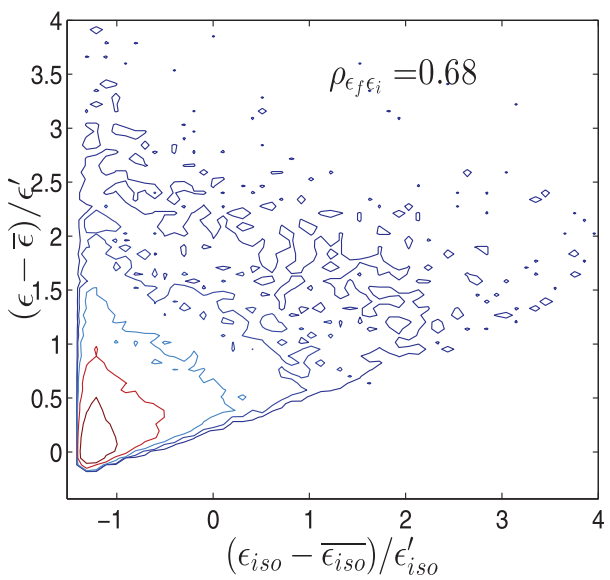

(a)

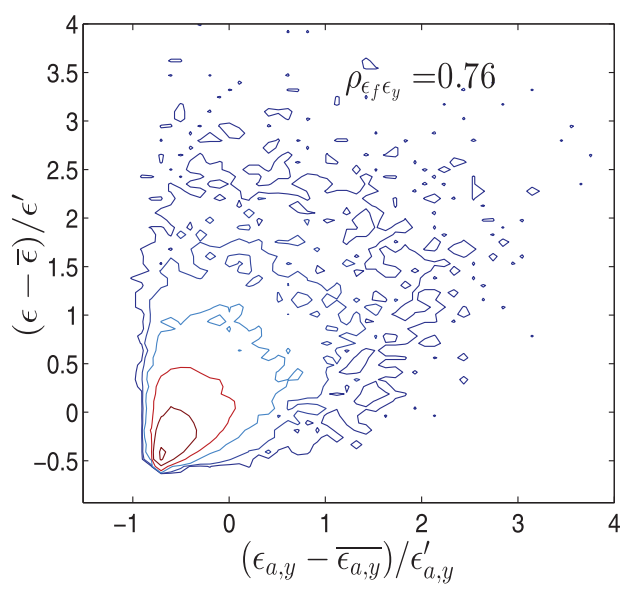

(c)

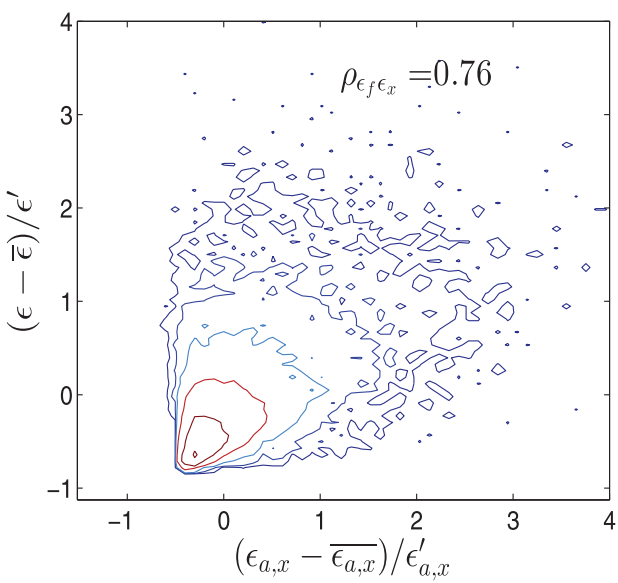

(b)

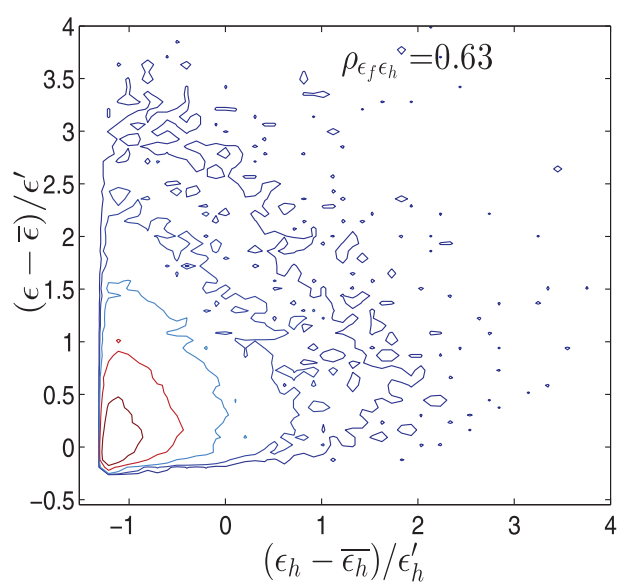

(d)

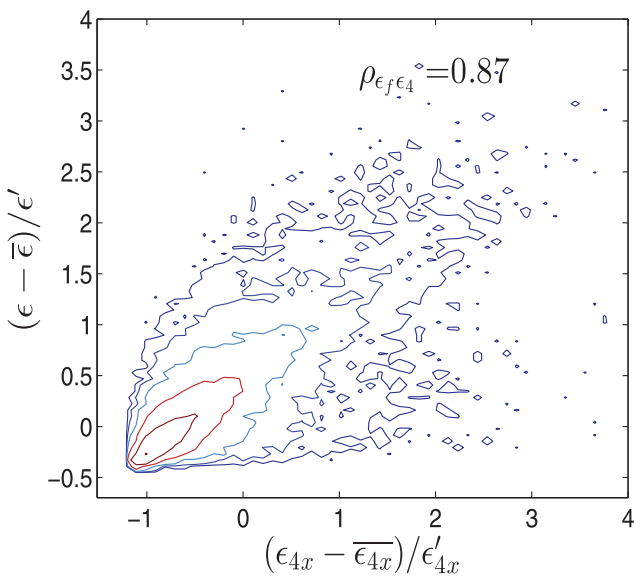

(e)

FIG. 12. Isocontours of JPDFs between the true $\epsilon$, Eq. (1), and different surrogates of $\epsilon$, Eqs. (2), (3), (5), (6), (9), at $y=0$ and $x / D=100$.

than $\rho_{\epsilon \epsilon_{i s o}}$ in spite of the fact that $\overline{\epsilon_{\text {hom }}}$ approximates $\bar{\epsilon}$ more closely than $\overline{\epsilon_{i s o}}$. A possible cause for this is the influence of the instantaneous pressure on $\epsilon$ in the intermediate wake. Consequently, the hypothesis of homogeneity for the velocity field may not be appropriate in the present flow. Clearly, this issue needs to be investigated further in the view of the approximation $\epsilon_{\text {hom }}=\nu \omega^{2}$. This is 
TABLE II. Correlation coefficient $\rho_{\omega^{2} \epsilon_{i}}, \rho_{\omega^{2} \epsilon_{f}}$, and $\rho_{\omega^{2} \epsilon_{4 x}}$ at $x / D=100$.

\begin{tabular}{lcc}
\hline \hline & Present data & $C C^{13}$ \\
\hline$\rho_{\omega^{2} \epsilon_{i s o}}$ & 0.43 & 0.22 \\
$\rho_{\omega^{2} \epsilon}$ & 0.63 & 0.4 \\
$\rho_{\omega^{2} \epsilon_{4 x}}$ & 0.78 & $\cdots$ \\
\hline \hline
\end{tabular}

highlighted by the presence of the term $\nabla^{2} p$ in expression (4). Nonetheless, the fact that all the coefficients exceed 0.60 underline a significant level of correlation between $\epsilon$ and all the surrogates that have been used, regardless of the lateral position in the wake.

The correlation coefficients $\rho_{\omega^{2} \epsilon_{i s o}} \rho_{\omega^{2} \epsilon_{f}}$, and $\rho_{\omega^{2} \epsilon_{4 x}}$ are reported in Table II for the location $x$ $=100 \mathrm{D}$ at the centreline and compared with those measured by $\mathrm{Zhu}{ }^{13}$ for a $C C$ wake. The correlation coefficient $\rho_{\omega^{2} \epsilon_{4 x}}$ is the largest and $\rho_{\omega^{2}, \epsilon_{i}}$ the smallest (0.43). It is interesting to note that $\rho_{\omega^{2}, \alpha}(\alpha$ representing $\epsilon$ or $\epsilon_{\text {iso }}$ ) is larger for $S q C$ than $C C$ (Table II). This suggests that pressure effects are likely to be less important in a $S q C$ wake compared to the $C C$ wake. The implication of this is that $\rho_{\epsilon \epsilon_{\text {hom }}}$ is likely to be even smaller for $C C$ than $S q C$.

\section{CONCLUSIONS}

The instantaneous turbulent kinetic energy dissipation rate, $\epsilon$, and its mean distributions, $\bar{\epsilon}$, in a wake of a square cylinder were investigated using numerical simulations based on the lattice Boltzmann method. Various expressions for approximating $\bar{\epsilon}$ and $\epsilon$, based on local isotropy $\left(\epsilon_{\text {iso }}\right)$, local axisymmetry along $x\left(\epsilon_{a, x}\right)$ and $y\left(\epsilon_{a, y}\right)$, local homogeneity $\left(\epsilon_{h o m}\right)$, and homogeneity in the transverse plane, $\left(\epsilon_{4 x}\right)$, were assessed as surrogates for $\epsilon$.

All the approximations for $\bar{\epsilon}$ agree well with the true $\bar{\epsilon}$ when the distance downstream of the cylinder is larger than about 40 diameters. Figure 8 indicates that compensation between the components of $\epsilon$ is important and explains the agreement between $\bar{\epsilon}_{i s o}$ and $\bar{\epsilon}$. Closer to the cylinder, the collapse is reasonable across the wake, except for $\bar{\epsilon}_{i s o}$ and $\bar{\epsilon}_{a, y}$, which depart from $\bar{\epsilon}$ in the region $0 \leq y / D \leq 1$.

The analysis is then extended to the instantaneous values of $\epsilon$ using PDFs and JPDFs. It is found that $\epsilon_{4 x}$ have the best correlation with $\epsilon$, while $\epsilon_{\text {iso }}$ and $\epsilon_{\text {hom }}$ exhibit the smallest correlation with $\epsilon$. Interestingly, $\epsilon_{i}$ so appears to slightly better surrogate for $\epsilon$ than $\epsilon_{\text {hom }}$ due to the non-negligible role of $\nabla^{2} p$ in Eq. (4).

A comparison of the present correlation coefficients between $\omega^{2}$ and $\epsilon$ with those obtained in a $C C$ by Zhu and Antonia, ${ }^{13}$ reveals that $S q C$ generates a more isotropic wake than $C C$. Consequently, the pressure effects are more important in a $C C$ wake compared to the $S q C$ wake. This explains why $\overline{\epsilon_{i s o}}$ is a better surrogate of $\bar{\epsilon}$ in the $S q C$ wake than in the $C C$ wake.

Finally, the results indicate that $\epsilon_{4 x}$ (Eq. (9)) should be an adequate surrogate for $\epsilon$ and can be used for correctly determining the probabilistic behaviour of $\epsilon$. This should be of significant value to experimentalist since one can then estimate reliably $\bar{\epsilon}$ and its instantaneous properties using a $4 X$-wire probe. ${ }^{13}$

${ }^{1}$ A. N. Kolmogorov, "The local structure of turbulence in incompressible viscous fluid for very large reynolds number," Dokl. Akad. Nauk SSSR 30, 301 (1941).

${ }^{2}$ A. N. Kolmogorov, "Dissipation of energy in the locally isotropic turbulence," Dokl. Akad. Nauk SSSR 32, 16 (1941), available at http://www.jstor.org/stable/51981.

${ }^{3}$ K. R. Sreenivasan and R. A. Antonia, "The phenomenology of small-scale turbulence," Annu. Rev. Fluid Mech. 29, 435-472 (1997).

${ }^{4}$ L. W. B. Browne, R. A. Antonia, and D. A. Shah, “Turbulent energy dissipation in a wake," J. Fluid Mech. 179, 307-326 (1987).

${ }^{5}$ G. Fabris, "Conditionally sampled turbulent thermal and velocity fields in the wake of a warm cylinder and its interaction with an equal cool wake," Ph.D. thesis, Illinois Institute of Technology, 1974.

${ }^{6}$ D. Aronson and L. Lofdahl, "The plane wake of a cylinder: Measurements and inferences on turbulence modeling," Phys. Fluids 5, 1433-1437 (1993). 
${ }^{7}$ G. I. Taylor, "Statistical theory of turbulence," Proc. R. Soc. London A 151, 422 (1935), available at http://www.jstor.org/stable/96557.

${ }^{8}$ B. R. Pearson, "Experiments on small-scale turbulence," Ph.D. thesis, Mechanical Engineering, University of Newcastle, 1999.

${ }^{9}$ J. Mi and R. A. Antonia, “Approach to local axisymmetry in a turbulent cylinder wake,” Exp. Fluids 48, $933-947$ (2010).

${ }^{10}$ W. George and H. Hussein, "Locally axisymmetric turbulence," J. Fluid Mech. 233, 1-23 (1991).

${ }^{11}$ H. J. Hussein, "Evidence of local axisymmetry in the small scales of a turbulent planar jet," Phys. Fluids 6, 2058 (1994).

${ }^{12} \mathrm{~F}$. Thiesset, "Exploration analytique et expérimentale des interactions cohérence-turbulence au sein d'un écoulement de sillage,” Ph.D. thesis, Faculté des Sciences and Techniques de l'Université de Rouen, 2011.

${ }^{13}$ Y. Zhu and R. Antonia, "On the correlation between enstrophy and energy dissipation rate in a turbulent wake," Appl. Sci. Res. 57, 337-347 (1997).

${ }^{14}$ R. Antonia, P. Orlandi, and T. Zhou, "Assessment of a three-component vorticity probe in decaying turbulence," Exp. Fluids 33, 384-390 (2002).

${ }^{15}$ T. Zhou, Z. Hao, L. P. Chua, and Y. Zhou, "Comparisons between different approximations to energy dissipation rate in a self-preserving far wake," Phys. Rev. E 74, 056308 (2006).

${ }^{16}$ L. Djenidi, "Structure of a turbulent crossbar near-wake studied by means of lattice Boltzmann simulation," Phys. Rev. E 77, 036310 (2008).

${ }^{17}$ Z. Hao, T. Zhou, L. Chua, and S. Yu, "Approximations to energy and temperature dissipation rates in the far field of a cylinder wake,” Exp. Therm. Fluid Sci. 32, 791-799 (2008).

${ }^{18}$ R. A. Antonia, T. Zhou, and Y. Zhu, "Three-component vorticity measurements in a turbulent grid flow,” J. Fluid Mech. 374, 29-57 (1998).

${ }^{19}$ F. Thiesset, L. Danaila, and R. A. Antonia, "Dynamical effect of the total strain induced by the coherent motion on local isotropy in a wake," J. Fluid Mech. 720, 393-423 (2013).

${ }^{20}$ L. D. Landau and E. M. Lifshitz, Fluid Mechanics (Pergamon Press, London, 1959).

${ }^{21}$ U. Frisch, B. Hasslacher, and Y. Pomeau, "Lattice-gas automata for the Navier-Stokes equation," Phys. Rev. Lett. 56, 14 (1986).

${ }^{22}$ S. Chen and G. D. Doolen, "Lattice Boltzmann method for fluid flows," Annu. Rev. Fluid Mech. 30, 329-364 (1998)

${ }^{23}$ S. Succi, The Lattice Boltzmann Equation: For Fluid Dynamics and Beyond (Oxford University Press, 2001).

${ }^{24}$ L.-S. Luo, W. Liao, X. Chen, Y. Peng, and W. Zhang, "Numerics of the lattice Boltzmann method: Effects of collision models on the lattice Boltzmann simulations," Phys. Rev. E 83(5), 056710 (2011).

${ }^{25} \mathrm{D}$. Nie, J. Lin, and L. Qiu, "Direct numerical simulations of the decaying turbulence in rotating flows via the MRT-lattice Boltzmann method," Int. J. Comput. Fluid Dyn. 27(3), 173-183 (2013).

${ }^{26}$ L. Djenidi, "Lattice Boltzmann simulation of grid-generated turbulence," J. Fluid Mech. 552, 13-35 (2006).

${ }^{27}$ P. Burattini, P. Lavoie, A. Agrawal, L. Djenidi, and R. A. Antonia, "Power law of decaying homogeneous isotropic turbulence at low Reynolds number," Phys. Rev. E 73, 066304 (2006).

${ }^{28}$ O. Ertunc, N. O. Zyilmaz, H. Lienhart, F. Durst, and K. Beronov, "Homogeneity of turbulence generated by static-grid structures," J. Fluid Mech. 654, 473-500 (2010).

${ }^{29}$ L. Djenidi, S. F. Tardu, and R. A. Antonia, "Relationship between temporal and spatial averages in grid turbulence," J. Fluid Mech. 730, 593-606 (2013).

${ }^{30}$ L. Djenidi and R. A. Antonia, "Transport equation for the mean turbulent energy dissipation rate in low- $R_{\lambda}$ grid turbulence," J. Fluid Mech. 747, 288-315 (2014).

${ }^{31}$ L. Djenidi, S. Tardu, R. Antonia, and L. Danaila, "Breakdown of Kolmogorov's first similarity hypothesis in grid turbulence," J. Turbul. 15(9), 596-610 (2014).

${ }^{32}$ H. Abe, R. Antonia, and H. Kawamura, "Correlation between small-scale velocity and scalar fluctuations in a turbulent channel flow," J. Fluid Mech. 627, 1-32 (2009).

${ }^{33}$ B. E. Lee, "The effect of turbulence on the surface pressure field of a square prism," J. Fluid Mech. 69, 263-282 (1975).

${ }^{34}$ A. Okajima, "Strouhal numbers of rectangular cylinders," J. Fluid Mech. 123, 379-398 (1982).

${ }^{35}$ D. F. G. Durao, M. V. Heitor, and J. C. F. Pereira, "Measurements of turbulent and periodic flows around a square cross-section cylinder," Exp. Fluids 6, 298-304 (1988).

${ }^{36}$ A. Sohankar, C. Norberg, and L. Davidson, "Simulation of three-dimensional flow around a square cylinder at moderate Reynolds numbers," Phys. Fluids 11, 288 (1999).

${ }^{37}$ Y. Kaneda, T. Ishihara, M. Yokokawa, K. Itakura, and A. Uno, "Energy dissipation rate and energy spectrum in high resolution direct numerical simulations of turbulence in a periodic box," Phys. Fluids 15(2), L21-L24 (2003).

${ }^{38}$ P. Parnaudeau, J. Carlier, D. Heitz, and E. Lamballais, "Experimental and numerical studies of the flow over a circular cylinder at Reynolds number 3900," Phys. Fluids 20, 085101 (2008).

${ }^{39}$ A. G. Kravchenkoa and P. Moin, "Numerical studies of flow over a circular cylinder at Re=3900," Phys. Fluids 12, 403-417 (2000).

${ }^{40}$ R. Antonia, H. Abe, and H. Kawamura, "Analogy between velocity and scalar fields in a turbulent channel flow," J. Fluid Mech. 628, 241-268 (2009).

${ }^{41}$ Hiroyuki Abe, private communication (2009). 